\title{
Network-Structured Firefly Algorithm and its Behavior
}

\author{
Haruna Matsushita ${ }^{\dagger}$, Daiki Matsumoto and Yoshifumi Nishio $\ddagger$ \\ $\dagger$ Department of Electronics and Information Engineering, Kagawa University \\ Email: haruna@eng.kagawa-u.ac.jp \\ $\ddagger$ Department of Electrical and Electronic Engineering, Tokushima University \\ Email: nishio@ee.tokushima-u.ac.jp
}

\begin{abstract}
This paper proposes a network-structured firefly algorithm (NS-FA). The standard firefly algorithm (FA) consists of multiple fireflies. An attractiveness of the firefly is proportional to its brightness, and for any two fireflies, the less brighter one will be attracted by the brighter one at every generation step. However, the fireflies of the NS-FA have a network structure that changes with generation step, and they move depending on its network structure. That is to say, the firefly of the NS-FA is not affected by brighter firefly if there is no connection between the two fireflies. In other words, even if there is brighter firefly in the firefly swarm, the firefly is not always attracted to the brighter firefly. We apply the NS-FA to various optimization benchmarks and confirm its effectiveness.
\end{abstract}

\section{Introduction}

Nature-inspired algorithms are among the most powerful algorithms for optimization. Among these biology-derived algorithms, the multi-agent metaheuristic algorithms such as the particle swarm optimization (PSO) [1] are hot research topics. The firefly algorithm (FA) [2] is an optimization algorithm based on a swarm intelligence as PSO.

The FA is inspired by the flashing behavior of fireflies. The primary purpose for a firefly's flash is to act as a signal system to attract other fireflies. In this study, we propose a network-structured firefly algorithm (NS-FA). The most important feature of the NS-FA is that fireflies of the NS-FA have network structure. In the standard FA, an attractiveness is proportional to their brightness, and for any two fireflies, the less brighter one will be attracted by the brighter one at every generation step. However, the firefly of the NS-FA is not affected by brighter firefly if there is no connection between the two fireflies. It is stochastically decided whether the fireflies of the NS-FA are connected with brighter fireflies and are disconnected from less brighter one. In other words, even if the brighter fireflies exist, the firefly is not always attracted to brighter one. We apply the NS-FA to various optimization benchmarks and compare the NS-FA with the PSO and the standard FA. Simulation results show that both the FAs can obtain better results than the PSO for the multimodal functions, and the NS-FA improves the optimization performance from the standard FA.
Objective function $f(\boldsymbol{x}), \boldsymbol{x}=\left(x_{1}, \cdots, x_{D}\right)^{T}$.

Initialize positions of fireflies $\boldsymbol{x}_{i}(i=1,2, \cdots, M)$.

Light intensity $I_{i}$ is determined by $f\left(\boldsymbol{x}_{i}\right), I_{i}=f\left(\boldsymbol{x}_{i}\right)$.

Generation step $t=0$.

while $\left(t<\right.$ MaxGeneration $\left.t_{\max }\right)$ do

for $i=1$ to $M$, all $M$ fireflies do

for $j=1$ to $M$, all $M$ fireflies do

if $I_{i}>I_{j}$ then

Move firefly $i$ toward $j$ according to Eq.(1).

end if

end for $j$

end for $i$

Evaluate new solutions $f\left(\boldsymbol{x}_{i}\right)$,

Rank the fireflies and find the current global best

$\boldsymbol{g}_{*}$.

end while

Figure 1: Pseudo code of the Firefly Algorithm (FA) for minimum optimization problems.

\section{Firefly Algorithm(FA)}

The FA is inspired by the flashing behavior of fireflies. The FA is assumed as follows: (1) All fireflies are unisex, so that one firefly will be attracted to all other fireflies. (2) Attractiveness is proportional to their brightness, and for any two fireflies, the less brighter one will be attracted by the brighter one. However, the brightness can decrease as their distance increases. If there are no fireflies brighter than a given firefly, it will move randomly. (3) The brightness of a firefly is affected or determined by the landscape of the objective function. For a minimum optimization problem $f(\boldsymbol{x})$ as this study, the light intensity $I_{i}$ of a firefly $i$ is determined by $I_{i}=f\left(\boldsymbol{x}_{i}\right)$.

Based on these three rules, the basic steps of the FA can be summarized as the pseudo code shown in Fig. 1. The initial positions of fireflies are generated at random $\left(\boldsymbol{x}_{i} \in\right.$ $\left[x_{\min }, x_{\max }\right]^{D}$ ).

The movement of a firefly $i$ is attracted by another more attractive firefly $j$, which has better solution, is determined 
by

$$
\boldsymbol{x}_{i}^{\text {new }}=\boldsymbol{x}_{i}^{\text {old }}+\beta_{i, j}\left(\boldsymbol{x}_{j}(t)-\boldsymbol{x}_{i}^{\text {old }}\right)+\alpha(t)\left(\operatorname{Random}()-\frac{1}{2}\right) L,
$$

where the second term is due to the attraction. The attractiveness $\beta$ is determined by

$$
\beta_{i, j}=\left(\beta_{0}-\beta_{\min }\right) e^{-\gamma r_{i, j}^{2}}+\beta_{\min },
$$

where $\beta_{0}$ is the attractiveness at $r=0, \beta_{\min }$ is the minimum value of $\beta$, and an absorption coefficient $\gamma$ is crucially important in determining the speed of the convergence. Thus, the attractiveness will vary with the distance $r_{i, j}$ between firefly $i$ and $j$;

$$
r_{i, j}=\left\|\boldsymbol{x}_{i}^{\mathrm{old}}-\boldsymbol{x}_{j}(t)\right\|=\sqrt{\sum_{d=1}^{D}\left(x_{i, d}-x_{j, d}\right)^{2}} .
$$

The third term of Eq. (1) is randomization with $\alpha(t)$ being the randomization parameter;

$$
\alpha(t)=\alpha(0)\left(1-\left(\frac{10^{-4}}{0.9}\right)^{1 / t_{\max }}\right),
$$

where $\operatorname{Random}()$ is a random number generator uniformly distributed in $[0,1]$, and $L$ is the average scale of the problem, $\left|x_{\max }-x_{\min }\right|$. The brightest firefly $k$ moves randomly according to

$$
\boldsymbol{x}_{k}(t+1)=\boldsymbol{x}_{k}(t)+\alpha(t)\left(\operatorname{Random}()-\frac{1}{2}\right) L .
$$

\section{Network-Structured Firefly Algorithm (NS-FA)}

We explain the NS-FA in detail. The most important feature of the NS-FA is that fireflies of the NS-FA have network structure. The firefly of the standard FA moves toward brighter fireflies. However, the fireflies of the NS-FA are attracted to only directly connected fireflies. Therefore, the firefly of the NS-FA is not affected by brighter firefly if there is no connection between the two fireflies. In addition, the network structure is changed with generation. The firefly of the NS-FA is stochastically connected with brighter fireflies and is disconnected from less brighter fireflies. The pseudo code of the NS-FA is shown in Fig. 2.

An initial network structure of the NS-FA is assumed as ring-topology. The connection among the fireflies is denoted by a connection matrix $C$. If the firefly $i$ is connected to the firefly $j, C_{i, j}=1$, otherwise, $C_{i, j}=0$.

The algorithm of the NS-FA is based on the standard FA. However, the fireflies of the NS-FA move depending on the network structure. A firefly $i$ is attracted to more attractive firefly $j$. However, if there is no connection between firefly $i$ and $j, i$ is not affected by $j$. Eq. (6) is the update equation of the NS-FA.

$$
\begin{aligned}
& \boldsymbol{x}_{i}^{\text {new }}= \\
& \begin{cases}\boldsymbol{x}_{i}^{\text {old }}+\beta\left(\boldsymbol{x}_{j}(t)-\boldsymbol{x}_{i}^{\text {old }}\right)+ & \alpha(t)\left(\operatorname{Random}()-\frac{1}{2}\right) L, \\
\boldsymbol{x}_{i}^{\text {old }}, & C_{i, j}=1 \text { AND } I_{i}>I_{j}\end{cases}
\end{aligned}
$$

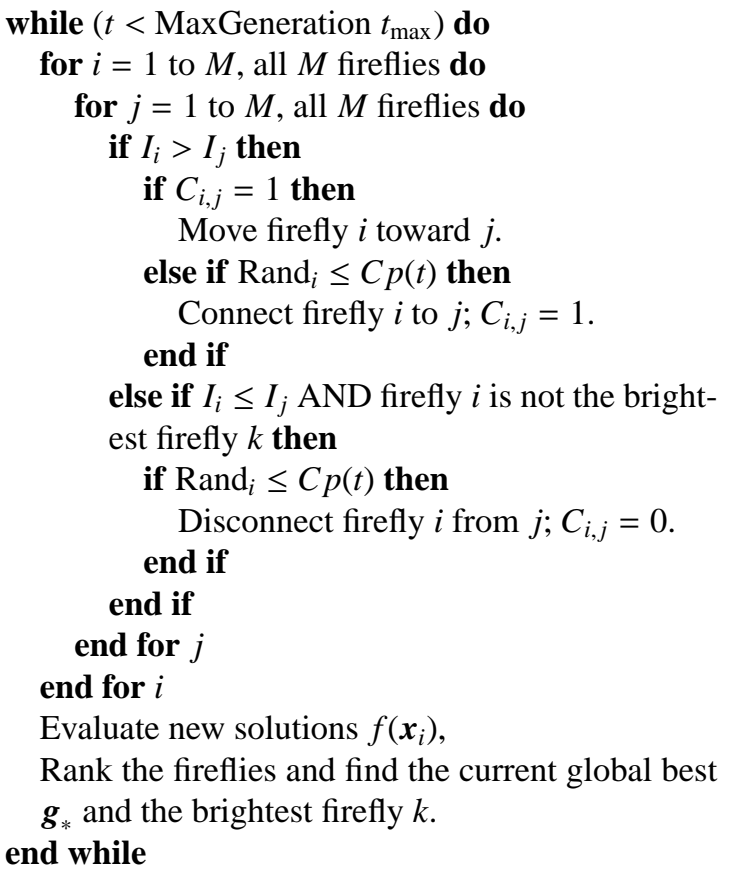

Evaluate new solutions $f\left(\boldsymbol{x}_{i}\right)$,

Rank the fireflies and find the current global best

$\boldsymbol{g}_{*}$ and the brightest firefly $k$.

end while

Figure 2: Pseudo code of the NS-FA for minimum optimization problems.

Next, we update connections between the firefly $i$ and others. The firefly $i$ is stochastically connected to the brighter fireflies. If $\operatorname{Rand}_{i} \leq C p(t), i$ is connected to $j$ according to

$$
C_{i, j}=1, \quad I_{i}>I_{j} .
$$

The firefly $i$, except the brightest firefly $k$, is stochastically disconnected from less brighter fireflies. If $\operatorname{Rand}_{i} \leq C p(t)$, $i$ is disconnected from $j$ according to

$$
C_{i, j}=0, \quad I_{i} \leq I_{j}, \quad i \neq k
$$

where $\operatorname{Rand}_{i}$ is a random number generator uniformly distributed in $[0,1]$ and $C p(t)$ is a connection probability determined by

$$
C p(t)=\frac{t}{t_{\max }} .
$$

By repeating these process, the network structure keeps on changing, and the fireflies converge to the optimum solution.

\section{Simulation}

We apply the NS-FA to 6 benchmark optimization problems summarized in Table 1. $f_{1}-f_{3}$ are unimodal functions, and $f_{4}-f_{6}$ are multimodal functions with numerous local minima. The optimum solution $\boldsymbol{x}^{*}$ of all the functions except $f_{2}$ are $[0,0, \ldots, 0]$ and for $f_{2}$ is $[1,1, \ldots, 1]$, and its optimum value $f\left(\boldsymbol{x}^{*}\right)$ is 0 . All the functions have $D$ variables, 
Table 1: Benchmark objective functions.

\begin{tabular}{llrl}
\hline Function name & Test Function & Initialization Space \\
\hline \hline Sphere; & $f_{1}(\boldsymbol{x})=\sum_{d=1}^{D} x_{d}^{2}$, & $x \in[-5.12,5.12]^{D}$ \\
Rosenbrock; & $f_{2}(\boldsymbol{x})=\sum_{d=1}^{D-1}\left(100\left(x_{d}^{2}-x_{d+1}\right)^{2}+\left(1-x_{d}\right)^{2}\right)$, & $x \in[-2.048,2.048]^{D}$ \\
$3^{\text {rd }}$ De Jong; & $f_{3}(\boldsymbol{x})=\sum_{d=1}^{D}\left|x_{d}\right|$, & & $x \in[-2.048,2.048]^{D}$ \\
Rastrigin; & $f_{4}(\boldsymbol{x})=\sum_{d=1}^{D}\left(x_{d}^{2}-10 \cos \left(2 \pi x_{d}\right)+10\right)$, & $x \in[-5.12,5.12]^{D}$ \\
Ackley; & $f_{5}(\boldsymbol{x})=\sum_{d=1}^{D-1}\left(20+e-20 e^{\left.-0.2 \sqrt{0.5\left(x_{d}^{2}+x_{d+1}^{2}\right)}-e^{0.5\left(\cos \left(2 \pi x_{d}\right)+\cos \left(2 \pi x_{d+1}\right)\right)}\right),}\right.$ & $x \in[-30,30]^{D}$ \\
Stretched V; & $f_{6}(\boldsymbol{x})$ & $=\sum_{d=1}^{D-1}\left(x_{d}^{2}+x_{d+1}^{2}\right)^{0.25}\left(1+\sin ^{2}\left(50\left(x_{d}^{2}+x_{d+1}^{2}\right)^{0.1}\right)\right)$, & \\
\hline
\end{tabular}

Table 2: Comparison results.

\begin{tabular}{c|c|ccc}
\hline$f$ & & PSO & FA & NS-FA \\
\hline \multirow{2}{*}{$f_{1}$} & Mean & $\mathbf{9 . 0 9 3} \times \mathbf{1 0}^{-\mathbf{1 4}}$ & $4.693 \times 10^{-4}$ & $7.043 \times 10^{-6}$ \\
& Min & $4.647 \times 10^{-19}$ & $4.027 \times 10^{-4}$ & $7.765 \times 10^{-7}$ \\
\hline \multirow{2}{*}{$f_{2}$} & Mean & $\mathbf{2 6 . 8 6}$ & 28.41 & 28.20 \\
& Min & 0.4426 & 27.12 & 26.64 \\
\hline \multirow{2}{*}{$f_{3}$} & Mean & $\mathbf{0 . 0 0 6 0 1 7}$ & 0.1272 & 0.05504 \\
& Min & $4.401 \times 10^{-8}$ & 0.07035 & 0.01203 \\
\hline \multirow{2}{*}{$f_{4}$} & Mean & 66.63 & 26.12 & $\mathbf{2 3 . 5 3}$ \\
& Min & 32.83 & 12.10 & 13.93 \\
\hline \multirow{2}{*}{$f_{5}$} & Mean & 107.0 & 8.213 & $\mathbf{3 . 1 7 4}$ \\
& Min & 10.31 & 0.7439 & 0.2124 \\
\hline \multirow{2}{*}{$f_{6}$} & Mean & 26.49 & 4.951 & $\mathbf{4 . 4 9 9}$ \\
& Min & 13.60 & 3.242 & 3.205 \\
\hline
\end{tabular}

in this study, $D=30$. The initialization space corresponds to the scale of the problem $\left[x_{\min }, x_{\max }\right]^{D}$.

The NS-FA is compared with the PSO and the standard FA. For all the optimization algorithms in all the simulations, the population size $M$ is set to $30(=D)$. For both FAs, the parameters are set as $\beta_{0}=1, \beta_{\min }=0.2, \gamma=1$ and $\alpha(0)=0.5$. For the PSO, the inertia weight $w$ and the acceleration coefficients $c$ are set as $w=0.729$ and $c_{1}=c_{2}=1.494$, respectively. These parameters are defined according to [1] and [2]. The maximum generation $t_{\max }$ are set to 1000 and 500 for the PSO and the FAs, respectively. Results are evaluated by average over 100 trials.

Table 2 summarizes the mean result $f\left(\mathrm{~g}^{*}\right)$, and the best result of the PSO, the standard FA and the proposed NS-FA, over 100 trials. We can see that in the unimodal functions $f_{1}-f_{3}$, the PSO obtained the best results. This is because that the PSO can quickly converge and the optimization speed, namely convergence speed, is important for the uni- modal functions which have few local optima. However, both the FAs also obtained enough results as the optimization result, and the results of the NS-FA were better than the standard FA. The proposed NS-FA improved the optimization performance from the standard FA by $98.49 \%$, $0.75 \%$ and $56.72 \%$ on $f_{1}, f_{2}$ and $f_{3}$, respectively. For this reason, in the late stage of learning, the firefly of the NS-FA is connected to only brightest firefly $k$. In other words, the firefly of the NS-FA is affected by only $k$. By this effect, the fireflies of the NS-FA can converge more quickly than the standard FA.

In the results of the multimodal functions $f_{4}-f_{6}$, both FAs significantly improved the results from the PSO although the total number of function evaluations of FAs is 500 which is much less than 1000 of the PSO. Furthermore, the proposed NS-FA improved the optimization performance from the standard FA by $9.92 \%, 61.35 \%$ and $9.14 \%$ on $f_{4}, f_{5}$ and $f_{6}$, respectively. The network structure of the NS-FA changes with generation, and it products the diversity of the fireflies. Therefore, it is hard for the fireflies of the NS-FA to be trapped into the local optima. From these results, we can conclude that the NS-FA is effective for the multimodal problems than the standard FA and the PSO.

Next, we investigate the changes of connection relationship of NS-FA and the behavior of the fireflies to confirm the improvement of diversification of NS-FA. Figures 3 and 4 show the convergence process for Ackley's function of 30 fireflies in the conventional FA and NS-FA, respectively. From these figures, we can see that the fireflies of NS-FA are scattered over wider range than that of FA. Although the fireflies of FA tend to quickly converge in the early stages, the fireflies of NS-FA slowly converge. This is because the fireflies of NS-FA are not necessarily connected with the 


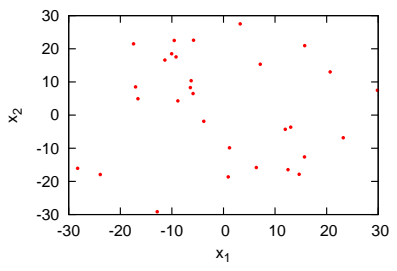

(a)

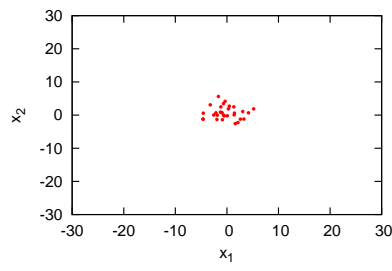

(b)

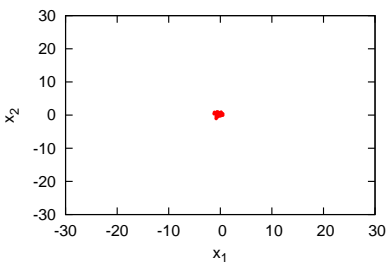

(c)

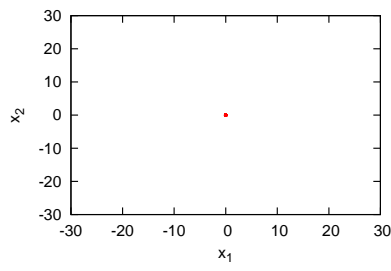

(d)

Figure 3: Convergence process of FA for Ackley's function. (a) $t=0$ (b) $t=10$ (c) $t=100$ (d) $t=500\left(=t_{\max }\right)$

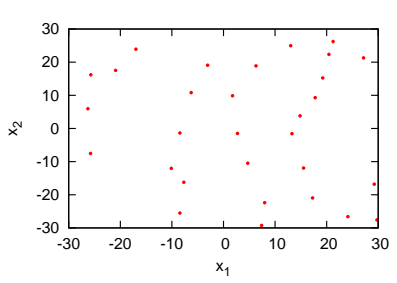

(a)

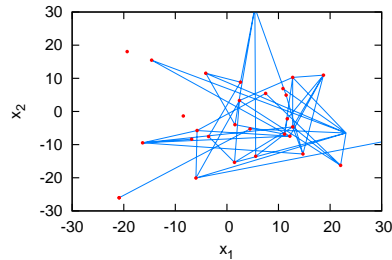

(b)

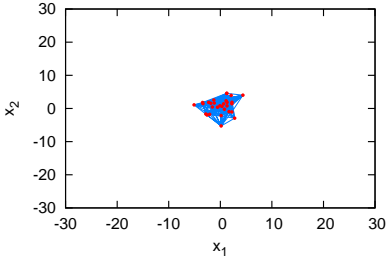

(c)

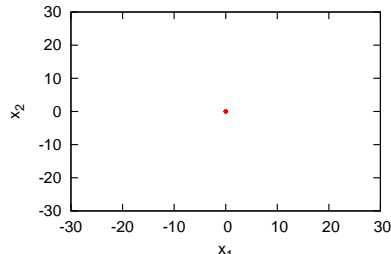

(d)

Figure 4: Convergence process of NS-FA for Ackley's function. (a) $t=0$ (b) $t=10$ (c) $t=100$ (d) $t=500\left(=t_{\text {max }}\right.$ )

best firefly, and the power of influence of the brighter firefly to others is not so much, as shown in Fig. 5(a) which is a magnified figure of Fig. 4. However, in the rate stages, NSFA converges to the optimum solution. This is because all the fireflies receive only the influence of the brightest firefly (Fig. 5(b)) and the local search was strengthened. From these results, we can conclude that because the behavior and convergence speed of the fireflies of NS-FA change with the iteration, its diversity was improved from FA.

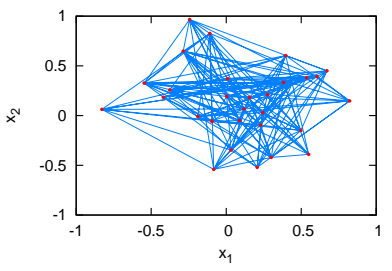

(a)

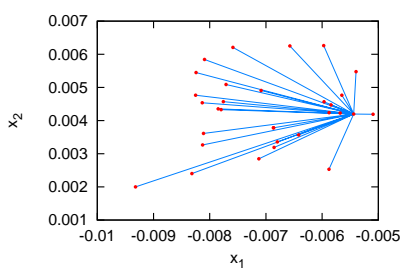

(b)
Figure 5: Connection relationship of NS-FA. (a) $t=200$. (b) $t=500$.

\section{Conclusions}

We have proposed the novel FA, the NS-FA. In the standard FA, an attractiveness is proportional to their brightness, and for any two fireflies, the less brighter one will be attracted by the brighter one at every generation step. However, the firefly of the NS-FA is not affected by brighter firefly if there is no connection between the two fireflies. In other words, even if there is brighter firefly, the firefly is not always attracted to brighter firefly.

We have applied the NS-FA to various optimization benchmarks and have compared the NS-FA with the PSO and the standard FA. From simulation results, for the unimodal functions, the PSO can obtain the best results among three algorithms. Both the FAs obtained the enough results as the optimization results although they are worse than the PSO.

For the multimodal functions, both the FAs obtained better results than the PSO. In addition, the NS-FA improved the optimization performance from the standard FA. From these results, the NS-FA is effective for the multimodal problems than the standard FA and the PSO.

\section{Acknowledgment}

This work was supported by KAKENHI 24700226.

\section{References}

[1] J. Kennedy and R. C. Eberhart, "Particle swarm optimization," in Proc. of IEEE. Int. Conf. on Neural Netw., pp. 1942-1948, 1995.

[2] Yang. X. S., "Nature-Inspired Metaheuristic Algorithms", Luniver Press, 2008. 